Recherches en histoire de l'art, histoire des civilisations, archéologie, anthropologie et muséologie

$6 \mid 2015$

Cahiers 6

\title{
Censure, éthique et propagande au sein de la revue URSS en construction
}

Censorship, ethics and propaganda in the magazine URSS en construction

\author{
Jessica Watson
}

\section{(2) OpenEdition}

Journals

Édition électronique

URL : http://journals.openedition.org/cel/321

DOI : $10.4000 /$ cel.321

ISSN : 2262-208X

Éditeur

École du Louvre

\section{Référence électronique}

Jessica Watson, «Censure, éthique et propagande au sein de la revue URSS en construction », Les Cahiers de l'École du Louvre [En ligne], 6 | 2015, mis en ligne le 01 avril 2015, consulté le 17 septembre 2019. URL : http://journals.openedition.org/cel/321 ; DOI : 10.4000/cel.321

\section{(9) $\odot \Theta \Theta$}

Les Cahiers de l'École du Louvre sont mis à disposition selon les termes de la licence Creative Commons Attribution - Pas d'Utilisation Commerciale - Pas de Modification 4.0 International. 


\title{
Censure, éthique et propagande au sein de la revue URSS en construction
}

\author{
Jessica Watson
}

\begin{abstract}
"Afin d'empêcher nos ennemis de l'intérieur comme de l'extérieur de l'Union soviétique de la possibilité de déformer et de discréditer le langage verbal et mathématique, nous avons décidé de nous tourner vers le dessin par la lumière (svetopis), le travail du soleil, c'est à dire la photographie'. "
\end{abstract}

URSS en construction, 1930.

Cette citation tirée d'un numéro de la revue SSSR na stroike (URSS en construction) résume parfaitement le rôle et les enjeux qui vont être ceux de la revue tout au long de sa parution, de 1929 à $1941^{2}$. Conçue pour être l'ambassadrice des valeurs et des avancées technologiques et industrielles de l'Union Soviétique, la revue, initialement publiée en russe, va très rapidement être traduite dans plusieurs langues et exportée dans les grandes puissances européennes: en France, en Angleterre, en Allemagne mais également en Espagne ${ }^{3}$. Créée au départ comme simple supplément à Nashi dostijenia ${ }^{4}$ (Nos réalisations), magazine littéraire populaire, la revue URSS en construction devient en peu de temps une publication photographique autonome où l'image prend l'ascendant sur le mot. Staline, qui souhaite rendre le magazine accessible au plus grand nombre de personnes possible, voit dans la photographie le moyen le plus efficace et le plus rapide de faire de la propagande politique, industrielle mais aussi culturelle. De simples textes et descriptions ne suffisent plus ; il est nécessaire, pour illustrer la modernisation fulgurante du pays, de la capturer avec un outil qui incarne cette modernité : l’appareil photographique.

Aux commencements du magazine, afin de valoriser au mieux le patrimoine industriel de l'URSS, chaque numéro est dédié à une branche spécifique de l'industrie. Aux photographies de sites industriels tels que la fabrique Dzerjinky ou la fabrique Krasnaya Talka ${ }^{6}$ s'ajoutent de courts textes expliquant le fonctionnement et le rendement de chaque site, afin de donner au lecteur une vision d'ensemble des capacités de production du pays. Au fil des ans, URSS en construction se diversifie et aborde d'autres sujets que l'industrie, tels que le sport, le divertissement et les loisirs ${ }^{7}$. Quelques numéros hors-série paraissent également pour célébrer les grands événements de l'histoire de l'Union soviétique, notamment l'anniversaire de la Révolution et celui de Joseph Staline ${ }^{8}$. Pendant les années 1930 le magazine devient ainsi la vitrine du monde soviétique et de

1. URSS en construction, $1930, \mathrm{n}^{\circ} 1$, p. 3.

2. Bien que le dernier numéro d'URSS en construction date de 1949, la production s'arrête en 1941. Seul un numéro paraît en 1949.

3. Dès sa première année de parution en 1930, le magazine est traduit en français, anglais et allemand. Ce n'est qu'en 1938 qu'une édition espagnole voit le jour. Erika Wolf, « When photographs speak, to whom do they talk: the origins and audience of SSSR na stroike », Left History, vol. 6, n² 2, 1999, pp. 73-76.

4. E. Wolf, « Le statut de la photographie dans la revue URSS en construction » dans Kristian Feigelson (dir.), Caméra politique : cinéma et stalinisme, Paris, Presses Sorbonne Nouvelle, 2005, pp. 60-69.

5. La revue URSS en construction devient une publication indépendante en 1930, soit un an après sa création.

6. URSS en construction, 1930, $\mathrm{n}^{\circ} 2$, consacré à la capitale de l'industrie textile soviétique.

7. URSS en construction, 1934, nos 7-8, consacré à la culture physique et au sport en URSS ;

Ibidem, 1935, n 7, consacré aux montres, cycles et phonographes ; Ibid., 1938, n 1, consacré au cinéma soviétique.

8. URSS en construction, 1937, nos 9-10-11-12, consacré aux vingt ans de la grande révolution socialiste d'Octobre. 
ses vertus, délaissant souvent l'objectivité au profit d'un dithyrambe de son chef et sauveur.

La participation d'artistes soviétiques de renom tels que El Lissitzky ${ }^{9}$, Alexandre Rodchenko ${ }^{10}$ et de leurs femmes Sofia Küppers-Lissitzky et Varvara Stepanova également actives sur la scène artistique constructiviste des années 1930 provoque des bouleversements importants au sein de la publication ${ }^{11}$. En effet, délaissant la technique photographique pure, ils mettent le photomontage ${ }^{12}$ à l'honneur, donnant ainsi au magazine une direction artistique affirmée (fig. 1).
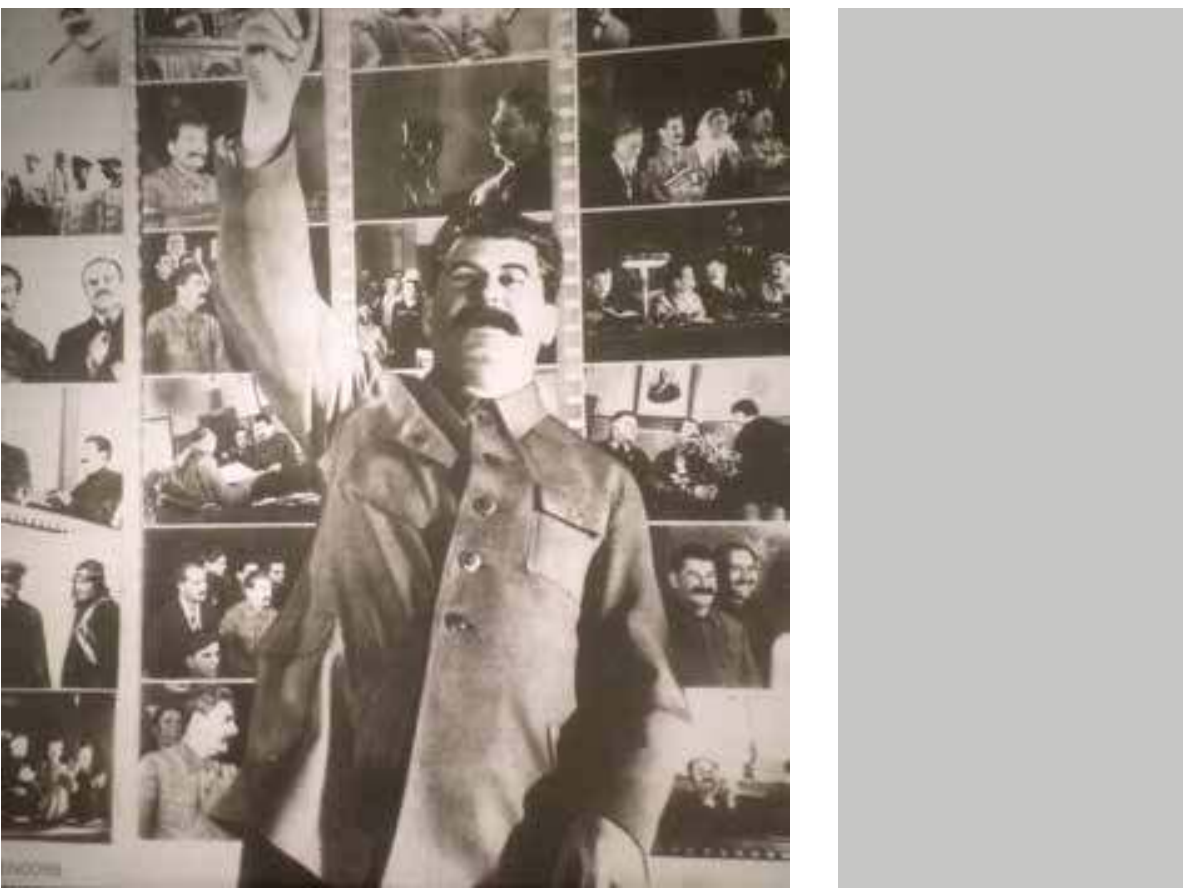

Les critiques soulevées par les historiens d'hier et d'aujourd'hui concernant les prises de position de ces artistes au sein d'URSS en construction ne semblent pas toujours prendre en considération la réalité du climat politique ${ }^{13}$. Ces derniers ont souvent été accusés de complicité avec le régime en raison de leur contribution à la promotion d'une image idéalisée et factice de l'Union soviétique, mais il faut se rappeler que sous le climat de terreur des années 1930, ceux qui s'opposent au régime n'y survivent pas. Toute œuvre qui viserait à critiquer le pouvoir, à le tourner en dérision ou qui ne le mettrait pas suffisamment en valeur est perçue comme une franche opposition au régime. La satire n'a pas sa place: elle est sanctionnée par l'emprisonnement, voire la condamnation à mort. Dimitri Chostakovitch par exemple, en 1936, deux ans après la création de son opéra Lady Macbeth de Mtsenk, est accusé d'être un ennemi du peuple et n'échappe à la mort qu' in extremis ${ }^{14}$. Vsevolod Meyerhold et Ossip Mendelstam n'auront pas

9. El Lissitzky commence à participer à la revue en 1932. Sa femme Sofia Küppers-Lissitzky contribue à certains numéros à partir de 1934 .

10. Rodchenko et sa femme Varvara Stepanova rejoignent le magazine un an plus tard en 1933. 11. En 1932, John Heartfield, artiste dada en visite en URSS, participe au dernier numéro de l'année de URSS en construction et introduit le photomontage. À partir de cette fructueuse collaboration, le photomontage prend une place prépondérante dans la conception artistique de la revue. Andrés Mario Zervigon, John Heartfield and the agitated image: photographie, persuasion, and the rise of avant-garde photomontage, Chicago, University of Chicago Press, 2012.

12. Le photomontage est la technique consistant à créer une image composite à partir de l'assemblage ou du collage de plusieurs photographies. Le résultat final peut être photographié afin de donner l'illusion qu'il s'agit originellement d'une seule et même photographie.

13. Annette Melot-Henry, La photographie soviétique de 1917 à 1945, Paris, Presses Universitaires de Paris 1, 2012, pp. 220-221.

14. Vladimir Chostakovitch (1906-1975), compositeur russe de renom, auteur d'opéras et de symphonies. Interrogé par le NKVD (Commissariat du peuple aux affaires intérieures), il devrait, selon diverses sources, sa survie à l'officier chargé de son dossier qui décida de ne pas poursuivre les chefs d'accusation. L'opéra Lady Macbeth du district de Mtsenk raconte I'histoire de Katerina Ismaïlova et de ses déboires dans la Russie du XIX siècle. Loin de promouvoir les valeurs de la famille soviétique chères à Staline, le dirigeant jugea l'opéra vulgaire, grotesque et le vit comme une ridiculisation directe du gouvernement. 
cette chance et seront tous deux exécutés ${ }^{15}$. Le sort de ces artistes illustre bien la répression qui sévit dans la sphère artistique. Lissitzky, Rodchenko et leurs épouses savent ce qu'ils encourent s'ils ne se conforment pas exactement aux règles établies par Staline concernant l'art. À partir de 1935, il n'est pas rare que des ouvrages encensés par la critique quelques années auparavant soient mis à l'index et détruits, comme Rodchenko en fera l'expérience. En 1934, ce dernier contribue à un ouvrage intitulé Dix ans d'Ouzbékistan ${ }^{16}$, mais en 1937, tandis que Staline mène une politique de purge parmi certains dirigeants ouzbeks, Rodchenko est contraint de caviarder les portraits de ces hommes devenus désormais des ennemis du peuple. "Cette défiguration, à laquelle Rodchenko a été contraint, n'est qu'un exemple parmi des milliers d'autres actes similaires perpétrés au moment de la Grande Terreur et après. Les bibliothèques de l'exUnion soviétique portent encore les cicatrices de ce vandalisme politique ${ }^{17}$. "

De manière indirecte, Rodchenko a vu les conséquences de la disgrâce. Forcé de mutiler son travail et d'altérer ses photographies originales, il sait mieux que quiconque la folie paranoïaque de Staline et ce qu'elle engendre. Le caviardage de certains portraits par Rodchenko a lieu en 1937, alors qu'il a déjà commencé à travailler occasionnellement pour URSS en construction ${ }^{18}$. Dans ces conditions, il n'est pas étonnant que Rodchenko s'en tienne aux limites artistiques qui lui sont imposées. De ce compromis dépend sa survie, tant artistique que physique.

De plus, les avantages liés à sa participation au magazine sont nombreux. Il bénéficie notamment d'une sécurité d'emploi et jouit d'une renommée qui reste plus ou moins intacte à l'étranger et qui croît à l'intérieur de l'Union Soviétique. URSS en construction constitue également une plateforme idéale pour poursuivre ses expériences de photomontages. Lui qui défend si ardemment la force et la simplicité des photographies de scènes quotidiennes ainsi que leur pouvoir de transmission aux masses, y voit l'opportunité de couvrir les grands événements du moment, d'être présent sur les lieux et de pouvoir exercer son art en toute sérénité sans craindre des représailles :

"Lissitzky and Rodchenko were caught in the contradiction of national development and ruthless politics just as was URSS in construction itself [...] In the face of eminent danger, Lissitzky's and Rodchenko's struggle for utopia became instead a striving for survival, and modest acts of resistance in an imperfect world became an acceptable end. And that can read more usefully as failed hope rather than tragedy ${ }^{19}$."

Les modestes actes de résistance auxquels Victor Margolin fait allusion ne sont pas très clairs. Il s'agit sans doute du refus des deux photographes de devenir des collaborateurs à plein temps au sein de la revue. Ayant préservé leur statut de photographes et d'artistes indépendants, ils ont encore la liberté de choisir les travaux auxquels ils souhaitent participer. Ils disposent de ce fait d'un certain

15. Vsevolod Meyerhold (1874-1940), dramaturge et écrivain russe. Refusant de se plier aux diktats de Staline qui ne voulait produire que des pièces de théâtre idéalistes et socialistes, il continue d'écrire des pièces constructivistes et futuristes. Arrêté en 1929, torturé et accusé d'espionnage et de trotskysme, il est exécuté, avec sa femme, en février 1940. Ossip Mendelstam (1891-1938), poète et essayiste russe. Soupçonné dès 1930 de conspirer contre le régime, il créé une poésie ouvertement contre le système et publie en 1933 la célèbre épigramme contre Staline Le Poignard du Kremlin. Il est arrêté un an plus tard et meurt de froid et de faim pendant son transfert d'un camp de travail à un autre à Kolyma.

16. David King, The commissar vanishes: the falsification of photographs and art in Stalin's Russia, New York, Metropolitan Books, 1997, p. 10. Traduit de I'anglais par Pascale Haas.

17. Idem, Ibidem, p. 10

18. Avant 1937, Rodchenko a participé à la publication de quatre numéros de la revue (URSS en construction, $1933, \mathrm{n}^{\circ} 12 ; 1935, \mathrm{n}^{\text {os }} 11,12 ; 1935, \mathrm{n}^{\circ} 12$ ).

19. « À I'instar de la revue URSS en construction elle-même, Lissitzky et Rodchenko furent pris entre deux feux : d'un côté le développement de leur pays et de l'autre une politique nationale impitoyable. [...] Confrontés à un danger réel, la lutte de Rodchenko et Lissitsky pour l'utopie se mua en une simple lutte de survie. Les plus petits actes de résistance dans ce monde imparfait parurent alors suffisants. Il est plus utile aujourd'hui d'y voir le signe d'un espoir déçu plutôt que d'une tragédie. » Victor Margolin, The Struggle for Utopia, Chicago, The University of Chicago Press, 1997, pp. 212-213. Traduction de l'auteur. 
pouvoir face au comité éditorial d'URSS en construction, puisque ce sont eux qui décident de diriger ou non la publication d'un numéro.

Il est indéniable que pendant cette période éthiquement floue, Rodchenko, Lissitzky et les autres artistes qui publient au sein d'URSS en construction ont produit des ouvres esthétiquement et techniquement époustouflantes qui ont bouleversées la scène artistique soviétique des années 1930. Sans URSS en construction, ces œuvres n'auraient sans doute jamais vu le jour. Ce photomontage tiré d'un numéro de la revue consacré au $15^{\mathrm{e}}$ anniversaire du Kazakhstan en est le témoignage même (fig. 2) ${ }^{20}$. Sur l'image, nous pouvons voir toute la maitrise de mise en page et de photomontage de Rodchenko et de Varvara Stepanova. Dans l'arrière-plan, apparait le paysage kazakh. Au-dessus sont apposées les dates importantes de l'histoire du peuple et, derrière le montage d'une photographie d'une sculpture monumentale de Lénine, un arrêté en russe indique les directives à appliquer sur le territoire kazakh. Le plan incliné en diagonale de Lénine ajoute de la profondeur à l'image et donne l'impression qu'il supervise les personnes en bas à gauche de l'image. Ces personnes tiennent des drapeaux imposants qui semblent s'élever sous la main droite de Lénine montrant la direction à suivre. Dans cette œuvre, Rodchenko adopte quatre angles de vue différents, créant ainsi une image composite qui comprend à la fois des sources littéraires, historiques et visuelles.
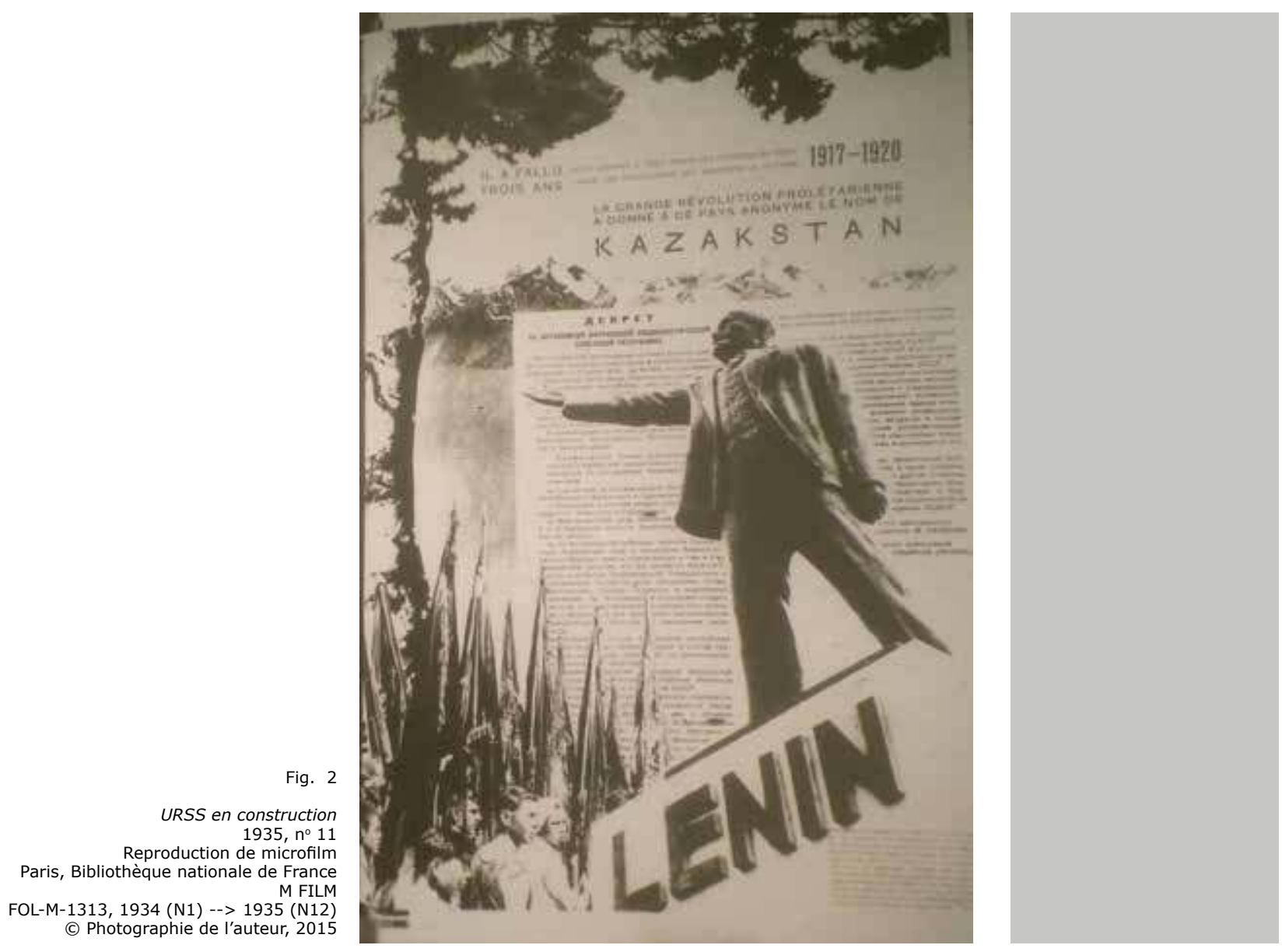

Ainsi, le contexte entourant la publication de ces photomontages et les questions d'éthique qu'elle soulève ne doivent pas occulter leurs qualités artistiques et esthétiques remarquables.

La publication du magazine durant cette période sombre, marquée par la répétition des purges et des procès ${ }^{21}$ pose inévitablement la question de la place de l'artiste, et notamment de son intégrité et de sa capacité à créer dans un tel

20. URSS en construction, 1935, $\mathrm{n}^{\circ} 11$

21. Hélène Carrère d'Encausse, Staline, I'ordre par la terreur, Paris, Flammarion, Champs, 1979, p. 69. 
ses vertus, délaissant souvent l'objectivité au profit d'un dithyrambe de son chef et sauveur.

La participation d'artistes soviétiques de renom tels que El Lissitzky ${ }^{9}$, Alexandre Rodchenko ${ }^{10}$ et de leurs femmes Sofia Küppers-Lissitzky et Varvara Stepanova également actives sur la scène artistique constructiviste des années 1930 provoque des bouleversements importants au sein de la publication ${ }^{11}$. En effet, délaissant la technique photographique pure, ils mettent le photomontage ${ }^{12}$ à l'honneur, donnant ainsi au magazine une direction artistique affirmée (fig. 1).
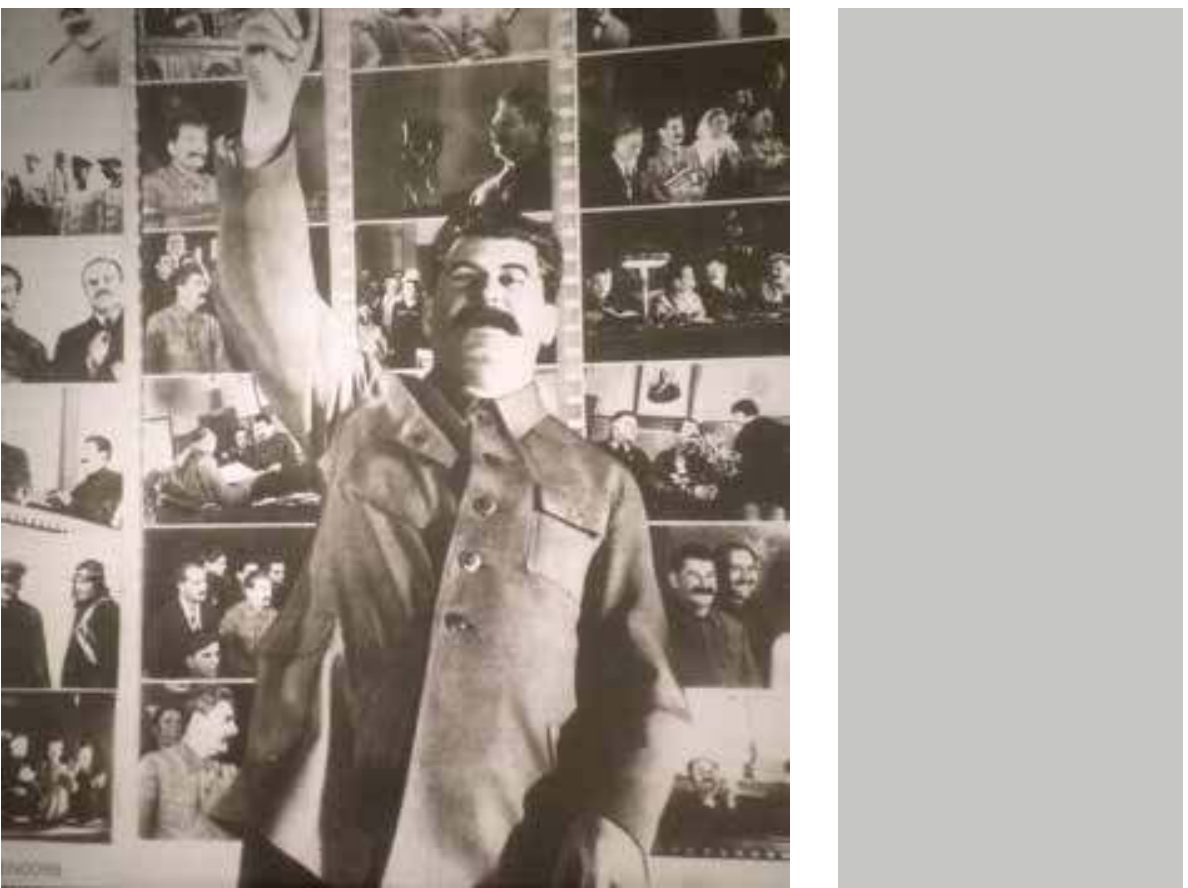

Les critiques soulevées par les historiens d'hier et d'aujourd'hui concernant les prises de position de ces artistes au sein d'URSS en construction ne semblent pas toujours prendre en considération la réalité du climat politique ${ }^{13}$. Ces derniers ont souvent été accusés de complicité avec le régime en raison de leur contribution à la promotion d'une image idéalisée et factice de l'Union soviétique, mais il faut se rappeler que sous le climat de terreur des années 1930, ceux qui s'opposent au régime n'y survivent pas. Toute œuvre qui viserait à critiquer le pouvoir, à le tourner en dérision ou qui ne le mettrait pas suffisamment en valeur est perçue comme une franche opposition au régime. La satire n'a pas sa place: elle est sanctionnée par l'emprisonnement, voire la condamnation à mort. Dimitri Chostakovitch par exemple, en 1936, deux ans après la création de son opéra Lady Macbeth de Mtsenk, est accusé d'être un ennemi du peuple et n'échappe à la mort qu' in extremis ${ }^{14}$. Vsevolod Meyerhold et Ossip Mendelstam n'auront pas

9. El Lissitzky commence à participer à la revue en 1932. Sa femme Sofia Küppers-Lissitzky contribue à certains numéros à partir de 1934 .

10. Rodchenko et sa femme Varvara Stepanova rejoignent le magazine un an plus tard en 1933. 11. En 1932, John Heartfield, artiste dada en visite en URSS, participe au dernier numéro de l'année de URSS en construction et introduit le photomontage. À partir de cette fructueuse collaboration, le photomontage prend une place prépondérante dans la conception artistique de la revue. Andrés Mario Zervigon, John Heartfield and the agitated image: photographie, persuasion, and the rise of avant-garde photomontage, Chicago, University of Chicago Press, 2012.

12. Le photomontage est la technique consistant à créer une image composite à partir de l'assemblage ou du collage de plusieurs photographies. Le résultat final peut être photographié afin de donner l'illusion qu'il s'agit originellement d'une seule et même photographie.

13. Annette Melot-Henry, La photographie soviétique de 1917 à 1945, Paris, Presses Universitaires de Paris 1, 2012, pp. 220-221.

14. Vladimir Chostakovitch (1906-1975), compositeur russe de renom, auteur d'opéras et de symphonies. Interrogé par le NKVD (Commissariat du peuple aux affaires intérieures), il devrait, selon diverses sources, sa survie à l'officier chargé de son dossier qui décida de ne pas poursuivre les chefs d'accusation. L'opéra Lady Macbeth du district de Mtsenk raconte I'histoire de Katerina Ismaïlova et de ses déboires dans la Russie du XIX siècle. Loin de promouvoir les valeurs de la famille soviétique chères à Staline, le dirigeant jugea l'opéra vulgaire, grotesque et le vit comme une ridiculisation directe du gouvernement. 
cette chance et seront tous deux exécutés ${ }^{15}$. Le sort de ces artistes illustre bien la répression qui sévit dans la sphère artistique. Lissitzky, Rodchenko et leurs épouses savent ce qu'ils encourent s'ils ne se conforment pas exactement aux règles établies par Staline concernant l'art. À partir de 1935, il n'est pas rare que des ouvrages encensés par la critique quelques années auparavant soient mis à l'index et détruits, comme Rodchenko en fera l'expérience. En 1934, ce dernier contribue à un ouvrage intitulé Dix ans d'Ouzbékistan ${ }^{16}$, mais en 1937, tandis que Staline mène une politique de purge parmi certains dirigeants ouzbeks, Rodchenko est contraint de caviarder les portraits de ces hommes devenus désormais des ennemis du peuple. "Cette défiguration, à laquelle Rodchenko a été contraint, n'est qu'un exemple parmi des milliers d'autres actes similaires perpétrés au moment de la Grande Terreur et après. Les bibliothèques de l'exUnion soviétique portent encore les cicatrices de ce vandalisme politique ${ }^{17}$. "

De manière indirecte, Rodchenko a vu les conséquences de la disgrâce. Forcé de mutiler son travail et d'altérer ses photographies originales, il sait mieux que quiconque la folie paranoïaque de Staline et ce qu'elle engendre. Le caviardage de certains portraits par Rodchenko a lieu en 1937, alors qu'il a déjà commencé à travailler occasionnellement pour URSS en construction ${ }^{18}$. Dans ces conditions, il n'est pas étonnant que Rodchenko s'en tienne aux limites artistiques qui lui sont imposées. De ce compromis dépend sa survie, tant artistique que physique.

De plus, les avantages liés à sa participation au magazine sont nombreux. Il bénéficie notamment d'une sécurité d'emploi et jouit d'une renommée qui reste plus ou moins intacte à l'étranger et qui croît à l'intérieur de l'Union Soviétique. URSS en construction constitue également une plateforme idéale pour poursuivre ses expériences de photomontages. Lui qui défend si ardemment la force et la simplicité des photographies de scènes quotidiennes ainsi que leur pouvoir de transmission aux masses, y voit l'opportunité de couvrir les grands événements du moment, d'être présent sur les lieux et de pouvoir exercer son art en toute sérénité sans craindre des représailles :

"Lissitzky and Rodchenko were caught in the contradiction of national development and ruthless politics just as was URSS in construction itself [...] In the face of eminent danger, Lissitzky's and Rodchenko's struggle for utopia became instead a striving for survival, and modest acts of resistance in an imperfect world became an acceptable end. And that can read more usefully as failed hope rather than tragedy ${ }^{19}$."

Les modestes actes de résistance auxquels Victor Margolin fait allusion ne sont pas très clairs. Il s'agit sans doute du refus des deux photographes de devenir des collaborateurs à plein temps au sein de la revue. Ayant préservé leur statut de photographes et d'artistes indépendants, ils ont encore la liberté de choisir les travaux auxquels ils souhaitent participer. Ils disposent de ce fait d'un certain

15. Vsevolod Meyerhold (1874-1940), dramaturge et écrivain russe. Refusant de se plier aux diktats de Staline qui ne voulait produire que des pièces de théâtre idéalistes et socialistes, il continue d'écrire des pièces constructivistes et futuristes. Arrêté en 1929, torturé et accusé d'espionnage et de trotskysme, il est exécuté, avec sa femme, en février 1940. Ossip Mendelstam (1891-1938), poète et essayiste russe. Soupçonné dès 1930 de conspirer contre le régime, il créé une poésie ouvertement contre le système et publie en 1933 la célèbre épigramme contre Staline Le Poignard du Kremlin. Il est arrêté un an plus tard et meurt de froid et de faim pendant son transfert d'un camp de travail à un autre à Kolyma.

16. David King, The commissar vanishes: the falsification of photographs and art in Stalin's Russia, New York, Metropolitan Books, 1997, p. 10. Traduit de I'anglais par Pascale Haas.

17. Idem, Ibidem, p. 10

18. Avant 1937, Rodchenko a participé à la publication de quatre numéros de la revue (URSS en construction, $1933, \mathrm{n}^{\circ} 12 ; 1935, \mathrm{n}^{\text {os }} 11,12 ; 1935, \mathrm{n}^{\circ} 12$ ).

19. « À I'instar de la revue URSS en construction elle-même, Lissitzky et Rodchenko furent pris entre deux feux : d'un côté le développement de leur pays et de l'autre une politique nationale impitoyable. [...] Confrontés à un danger réel, la lutte de Rodchenko et Lissitsky pour l'utopie se mua en une simple lutte de survie. Les plus petits actes de résistance dans ce monde imparfait parurent alors suffisants. Il est plus utile aujourd'hui d'y voir le signe d'un espoir déçu plutôt que d'une tragédie. » Victor Margolin, The Struggle for Utopia, Chicago, The University of Chicago Press, 1997, pp. 212-213. Traduction de l'auteur. 
pouvoir face au comité éditorial d'URSS en construction, puisque ce sont eux qui décident de diriger ou non la publication d'un numéro.

Il est indéniable que pendant cette période éthiquement floue, Rodchenko, Lissitzky et les autres artistes qui publient au sein d'URSS en construction ont produit des ouvres esthétiquement et techniquement époustouflantes qui ont bouleversées la scène artistique soviétique des années 1930. Sans URSS en construction, ces œuvres n'auraient sans doute jamais vu le jour. Ce photomontage tiré d'un numéro de la revue consacré au $15^{\mathrm{e}}$ anniversaire du Kazakhstan en est le témoignage même (fig. 2) ${ }^{20}$. Sur l'image, nous pouvons voir toute la maitrise de mise en page et de photomontage de Rodchenko et de Varvara Stepanova. Dans l'arrière-plan, apparait le paysage kazakh. Au-dessus sont apposées les dates importantes de l'histoire du peuple et, derrière le montage d'une photographie d'une sculpture monumentale de Lénine, un arrêté en russe indique les directives à appliquer sur le territoire kazakh. Le plan incliné en diagonale de Lénine ajoute de la profondeur à l'image et donne l'impression qu'il supervise les personnes en bas à gauche de l'image. Ces personnes tiennent des drapeaux imposants qui semblent s'élever sous la main droite de Lénine montrant la direction à suivre. Dans cette œuvre, Rodchenko adopte quatre angles de vue différents, créant ainsi une image composite qui comprend à la fois des sources littéraires, historiques et visuelles.
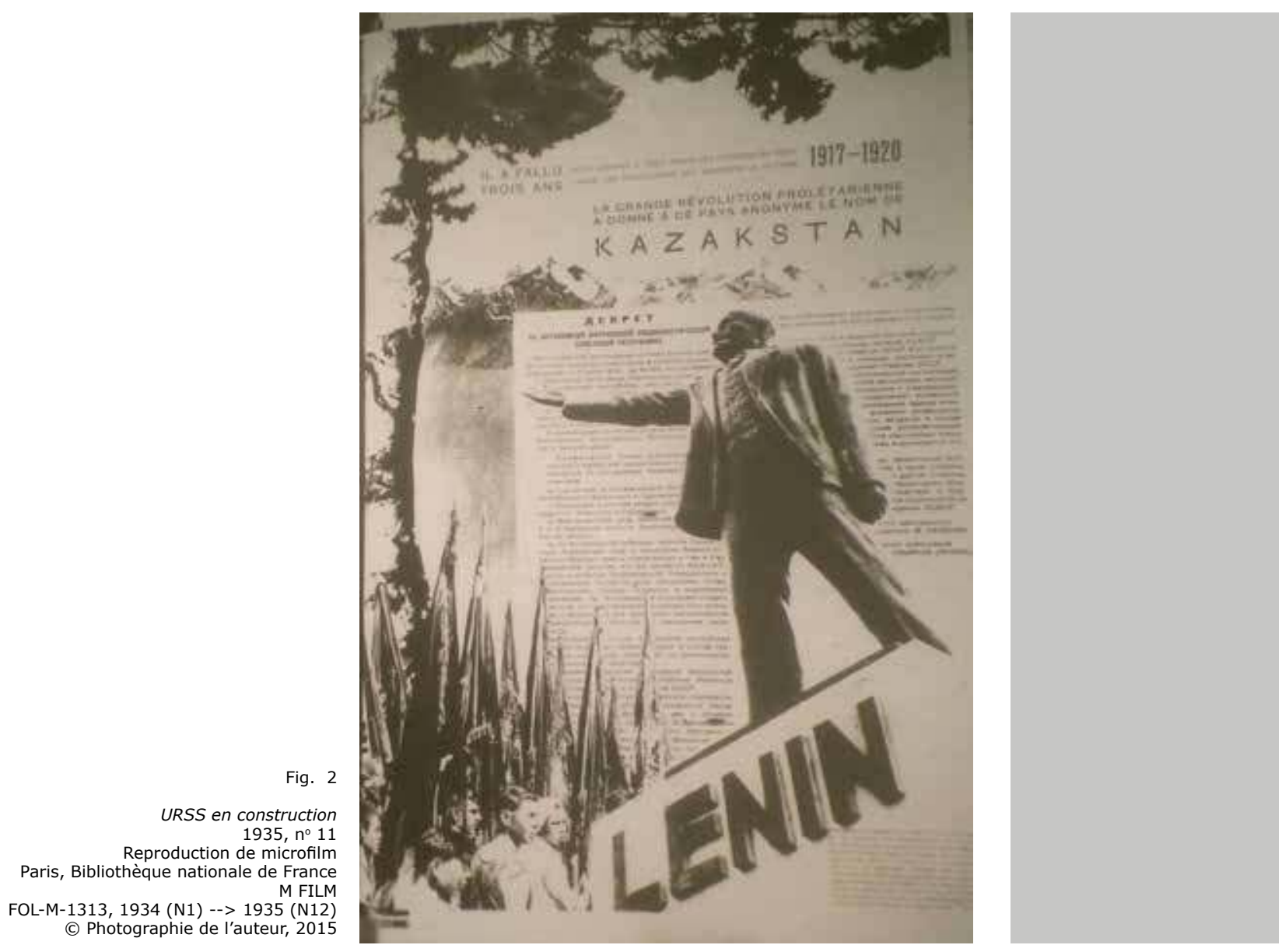

Ainsi, le contexte entourant la publication de ces photomontages et les questions d'éthique qu'elle soulève ne doivent pas occulter leurs qualités artistiques et esthétiques remarquables.

La publication du magazine durant cette période sombre, marquée par la répétition des purges et des procès ${ }^{21}$ pose inévitablement la question de la place de l'artiste, et notamment de son intégrité et de sa capacité à créer dans un tel

20. URSS en construction, 1935, $\mathrm{n}^{\circ} 11$

21. Hélène Carrère d'Encausse, Staline, I'ordre par la terreur, Paris, Flammarion, Champs, 1979, p. 69. 
contexte. Elle révèle a posteriori la dichotomie entre les faits et leur réécriture par l'Histoire officielle.

Tout d'abord, les années de publication de la revue constituent un moment paradoxal marqué à la fois par l'austérité du totalitarisme stalinien et par l'incroyable créativité qui caractérise alors la scène artistique en URSS, avec l'émergence de langages formels révolutionnaires ${ }^{22}$. Mais cette créativité n'est pas synonyme de vérité. Le monde tel qu'il est présenté dans URSS en construction diffère nettement de ce qui se passe de l'autre côté du miroir. Le manque de cohérence entre les faits présentés dans la revue et ceux qui ont véritablement lieu est de plus en plus flagrant et ne laisse aucun citoyen indifférent, remettant en cause la place des artistes qui contribuent à la revue. Au regard de leur travail photographique, il est difficile de ne pas s'interroger sur leur intégrité, tant le culte stalinien y est présent dans toute sa splendeur, sans pour autant écarter l'hypothèse que, par nécessité de survie et par volonté de distanciation, ces artistes se sont pris à leur propre jeu. Au nom de l'art, et plus précisément de leur art, ils prennent le risque d'entacher leurs convictions personnelles et de mettre leurs croyances politiques entre parenthèses.

Si Rodchenko et Lissitzky montrent une certaine loyauté au régime stalinien, ils continuent en parallèle à développer leur vision artistique dans l'idéologie constructiviste, même si celle-ci les amène à cautionner certaines atrocités. La présence de Rodchenko lors du chantier du Canal de la Mer Blanche est un des exemples les plus frappants de réécriture historique ${ }^{23}$. En effet, les ouvriers qui travaillent à la construction du barrage sont des criminels en réinsertion. Pour Staline, il s'agit de montrer que l'URSS permet aux hommes de faire table rase du passé et de se créer un nouvel avenir prometteur. En réalité, les conditions de travail et le climat déciment les travailleurs les uns après les autres. Lorsque Rodchenko entreprend son photoreportage, il ne capture que des visages souriants et enthousiastes, camouflant ainsi la réalité de ce qui se passe devant ses yeux. En omettant de photographier certains aspects et en retouchant certaines images, il contribue activement au mensonge visuel véhiculé par la revue. Comme l'écrit Erika Wolf :

"La transfiguration idéalisée de Rodchenko d'un rude camp de travail en colonie pénale progressiste à travers le médium de la photographie était une conséquence directe du constructivisme. Loin d'abandonner le constructivisme, Rodchenko utilisa URSS en construction pour continuer son travail visionnaire débuté auparavant ${ }^{24}$. "

On pourrait évoquer le célèbre projet de Lissitzky La tribune mobile de Lénine de $1920^{25}$ ou Le Monument à la Troisième Internationale de Vladimir Tatline de 1919-1920 (fig. 3) ${ }^{26}$. Bien que ces constructions ne voient jamais le jour, elles montrent l'utilisation du constructivisme au service du communisme. Le contexte de l'époque, nettement moins tendu, explique pourquoi ces œuvres ne sont pas soumises aux mêmes critiques virulentes que les travaux de Lissitzky et de Rodchenko pour URSS en construction, car ces œuvres célèbrent le régime avant les crimes et les atrocités des années 1930. Elles glorifient l'Union Soviétique et Lénine sans avoir pour but caché la dissimulation de la brutalité du gouvernement, puisqu'au début des années 1920, le régime n’a pas encore autant à rougir de ses

22. Voir Claude Leclanche-Boulé, Typographies et photomontages constructivistes, Paris, Papyrus, 1986.

23. URSS en construction, 1933, $\mathrm{n}^{\circ} 12$

24. E. Wolf, «The Visual Economy of Forced Labor », Picturing Russia, essays on visual evidence, New Haven, Yale University Press, 2008, p. 173, traduction de I'auteur.

25. El Lissitzky, Esquisse pour la Tribune mobile de Lénine, 1920, Galerie d'état Tretyakov, Moscou.

26. Vladimir Tatline, Maquette du Monument à la Troisième Internationale, 1919-1979, bois et métal, H. 4,20 ; D. 3,00 m, Paris, Musée National d'Art Moderne Centre Georges Pompidou, AM 1979-413. 
actes. Les travaux photographiques de Rodchenko et Lissitzky s'inscrivent donc dans la logique constructiviste entamée quelques années auparavant.
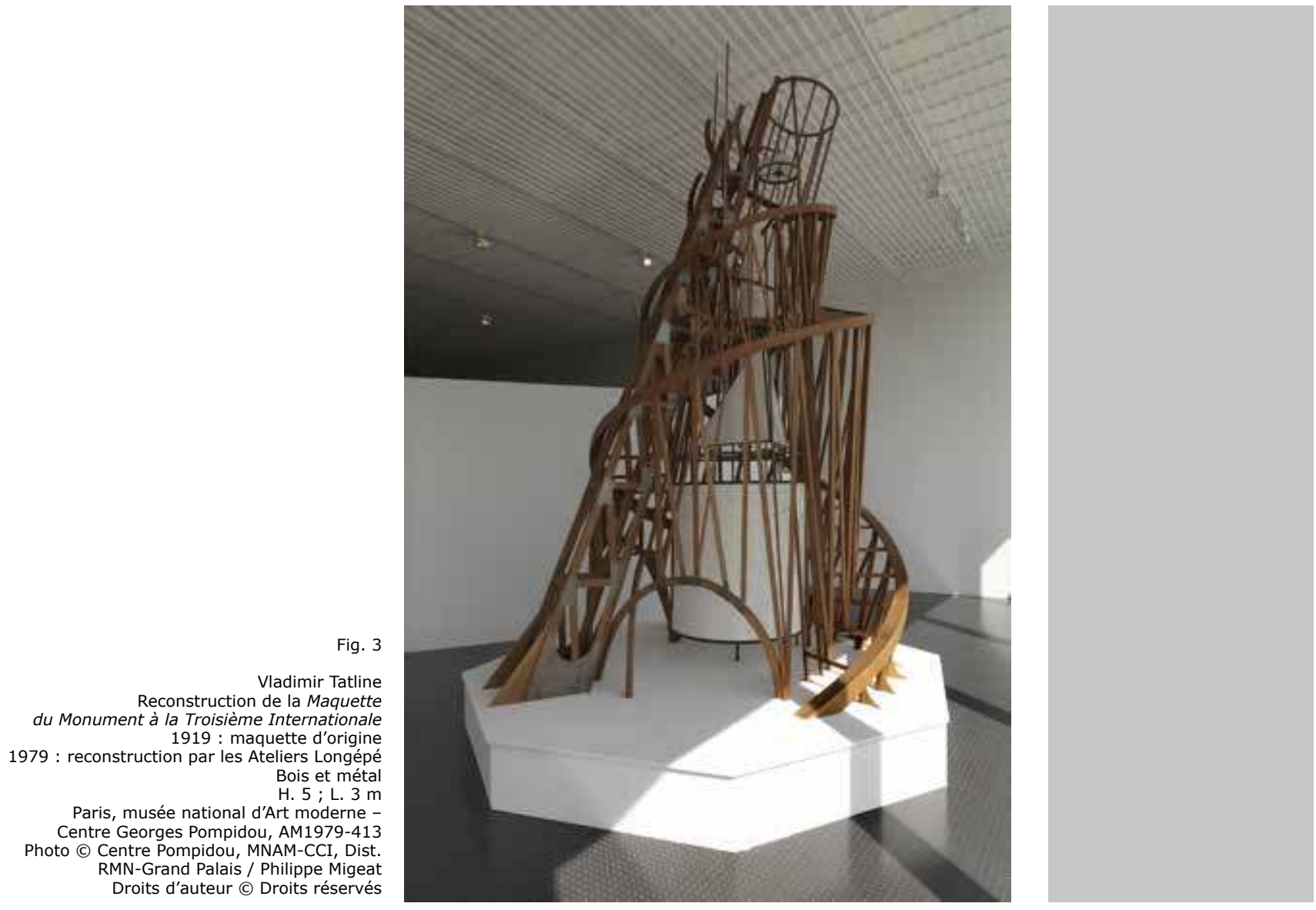

Les photomontages du magazine privilégient la géométrie des plans. À une composition trop chargée et visuellement saturée, les artistes préferent accentuer la verticalité ou l'horizontalité de ces plans. À la manière d'un mathématicien, les angles sont minutieusement pensés (fig. 4$)^{27}$ afin de maximiser l'espace de la page et d'insuffler un rythme et une tridimensionnalité qui transcendent les contraintes matérielles. On retrouve dans ces montages l'aspect fonctionnaliste des photographies dont le but est clairement celui d'une propagande visuelle, ainsi que les prémices de la factographie ${ }^{28}$, puisqu' une grande partie des montages combine avec élégance des photographies et des matériaux documentaires. Si le spectateur voit une image d'un chantier accompagné d'une carte géographique et d'un rapport de productivité signé par Staline lui-même, cela renforce l'impression initiale que l'image seule donnait au lecteur. Ainsi les photomontages d'URSS en construction sont des machines parfaitement maîtrisées qui, par leur composition irréprochable, veulent ôter tout doute quant à la véracité des informations qu'elles proposent à leur lectorat. On assiste à une mise en abyme où le texte fait partie de l'image qui, elle, répond au texte du magazine, contribuant ainsi à la cohésion et à l'homogénéité éditoriale de la revue.

Il est également important de considérer les moyens matériels mis à disposition des artistes d'URSS en construction. La revue étant presque intégralement subventionnée par le gouvernement, elle dispose d'un budget confortable pour ses reportages. Les artistes peuvent donc exercer leur art dans des conditions agréables, sans se soucier de manquer de moyens ou de matériel. Au regard de l'austérité qui règne ailleurs en URSS, il est aisé de comprendre la volonté

28. Comme son nom l'indique, le concept de factographie émerge d'une volonté de rajouter de la crédibilité aux informations véhiculées par les images. Voir Leah Dickerman, « The fact and the photograph », October, vol. 118, Automne 2006, pp. 142-145. 
d'Alpert, de Rodchenko et des autres artistes d'assurer leur survie matérielle et artistique.
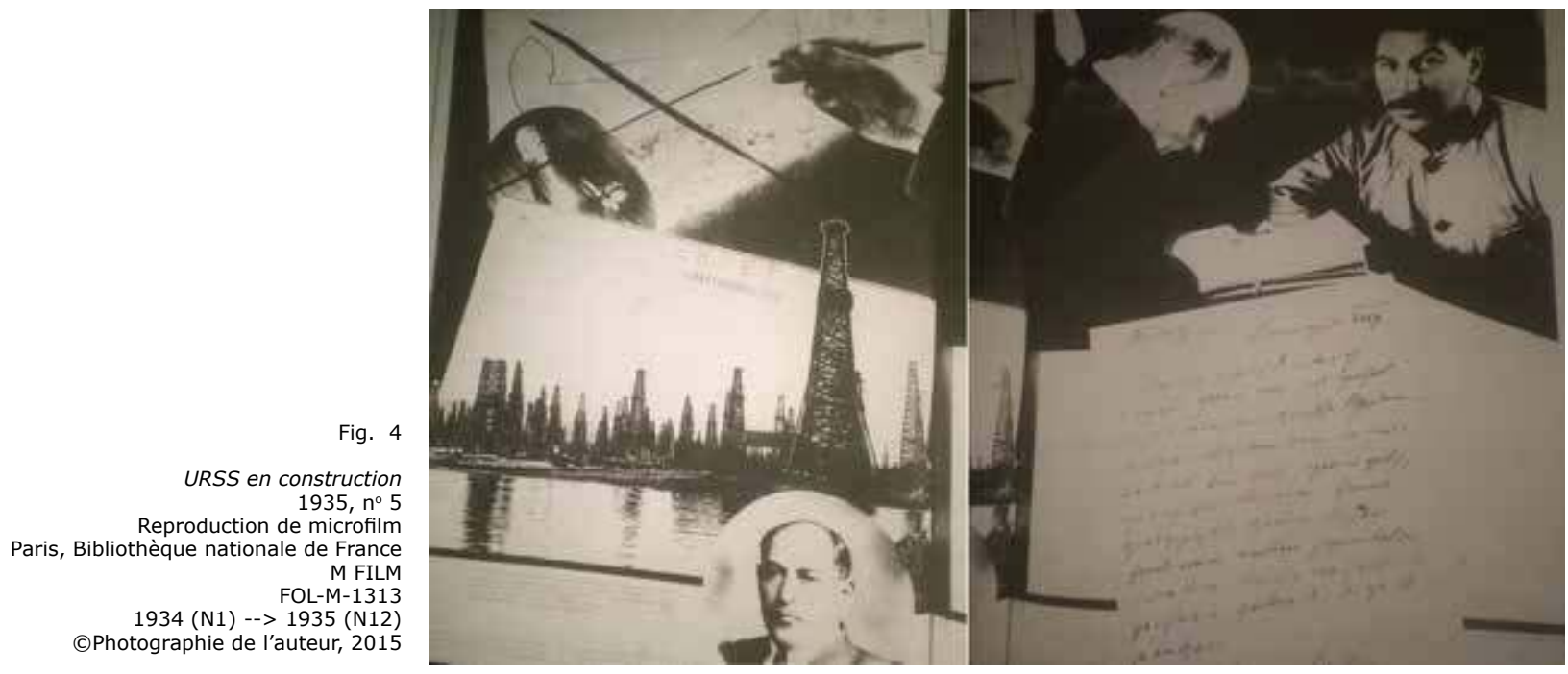

Ainsi, dans l'optique de faire un procès équitable aux photographes d'URSS en construction, il est impératif de prendre en compte les multiples aspects de la vie d'un artiste en Union Soviétique à cette période. La considération des moyens techniques n'atténue en rien le problème intrinsèque que pose la participation d'un artiste à un magazine d'État qui renvoie une fausse image de son pays ni la notion d'intégrité qui est si chère aux historiens de l'art lors de périodes historiquement et politiquement chargées. Indubitablement, Rodchenko, Lissitzky et leurs collègues au sein d'URSS en construction ne sont pas moralement irréprochables et ont indirectement contribué à répandre les mensonges tissés par Staline et ses hommes. Mais la valeur esthétique et technique d'une œuvre n'est pas dictée par le degré d'intégrité de l'artiste qui l'a produite. Ce sont des facteurs indépendants, qui nous permettent de réaffirmer aujourd'hui l'importance du corpus photographique d'URSS en construction et d'en relever ses qualités esthétiques indéniables qui fait de la revue un emblème du photomontage et de la mise en page avant-gardiste du début du $\mathrm{xx}^{\mathrm{e}}$ siècle, non seulement en URSS, mais également dans le reste de l'Europe.

Lexercice du photomontage, tel qu'il est pratiqué dans URSS en construction et qui implique la modification de certaines photographies ou images dans le but d'en proposer une nouvelle lecture, relève d'une démarche utopique qui vise à embellir ou améliorer le sujet ${ }^{29}$. Si par exemple certains personnages ne sont pas suffisamment visibles, il suffit d'agrandir la partie de la photographie où ils apparaissent et de la réintégrer à l'image originelle. Cette démarche découle donc d'un désir de perfectionner ce que l'on voit. Les années 1930 qui voient monter le réalisme socialiste sont empreintes de cette utopie, de ce que l'Union Soviétique veut et devrait être. Staline souhaite un État totalement orienté vers le communisme économique, politique et social, ce qui n'est évidemment pas faisable en si peu de temps. Le rêve ou l'utopie socialiste semble dominer les mentalités à cette période, car la société qui souffre de sa condition de vie difficile rêve d'un monde meilleur, celui que lui promet Staline. Il n'est donc pas surprenant de retrouver cette utopie dans le monde artistique, puisqu'à travers leurs œuvres, les artistes ont le pouvoir de transfigurer la réalité et de représenter un idéal imaginaire, personnel ou collectif. Mais, lorsque cet idéal sociétal s'éloigne de plus en plus et que les promesses de Staline ne sont pas tenues, l'utopie sur laquelle tous les espoirs se fondent disparaît et laisse place à une dystopie ${ }^{30}$ lourde à accepter.

29. Lutz Becker, Cut \& Paste, European Photomontage 1920-1945, Rome, Gangemi Editore, 2008, p. 6

30. Cat. d'exp., Utopia/Dystopia, construction and destruction in photography and collage, sous la direction de Yasufumi Nakamori, The Museum of Fine Arts, Houston, 11 mars-10 juin 2012, New Haven, Yale University Press, 2012. 
Pour les artistes d'URSS en construction, la prise de conscience est tout aussi douloureuse. Leur œuvre, qui devait participer à cette construction d'une ère nouvelle et meilleure pour tous, apparaît progressivement comme un mensonge destiné à leurrer le monde sur un régime qui ne cesse de se durcir, et ils doivent peu à peu se rendre à l'évidence que leur vision du communisme n'existera sans doute jamais. Leurs activités au sein d'URSS en construction à partir de 1935 ne font qu'accentuer cette déception. La réalité à laquelle ils sont confrontés lors de leurs photoreportages leur fait comprendre que le gouvernement souhaite mettre un épais vernis sur la vérité du quotidien soviétique. L'utopie s'arrête là où la retouche photographique commence.

Il est intéressant de constater que Rodchenko met fin à sa carrière de photomonteur en $1942^{31}$, peu de temps après l'arrêt de la publication d'URSS en construction pendant la Seconde Guerre mondiale. Tandis que certains y voient le simple désir de reprendre la peinture après avoir épuisé le médium photographique, il serait plus plausible d'y voir une rupture idéologique avec ce médium. Conscient, sans doute, du fossé qui existe entre le pouvoir transformatif de la photographie et la réalité non altérée par la pellicule, ainsi que son rôle dans l'élaboration d'une propagande mensongère, Rodchenko décide d'arrêter ses expérimentations techniques, impuissant face aux atrocités commises sous le régime. Sa perception de la photographie, immaculée à ses débuts, se trouve entachée par ce qu'il a photographié au fil des années, mais plus encore par ce qu'il n'a pas photographié par, ou par absence de, conscience professionnelle, selon le point de vue. La fin de la revue marque également la fin d'une période dans la carrière de Rodchenko. S'il continue néanmoins à organiser des expositions de photographies, auxquelles il ne participe pas, sa peinture à laquelle il se consacre à partir des années 1940 tend au contraire vers l'abstraction expressionniste, comme s'il rejetait désormais toute figuration ou toute notion de fait ${ }^{32}$.

Lissitzky, quant à lui, continue jusqu'à la fin de sa vie à produire des œuvres propagandistes pour l'Union Soviétique, mêlant photographie, typographie et design. Sa mort prématurée à l'âge de 51 ans ne nous permet pas de savoir vers quelle direction il aurait pu se tourner. Mais les liens beaucoup plus clairs et étroits de ce dernier avec le régime nous autorisent à stipuler qu'il n'aurait probablement pas abandonné la photographie ni le travail de propagande :

"Lissitzky showed his willingness to undertake any commission given to him; in fact, he became the Soviet Union's most knowledgeable designer of publications and exhibits during the 1930s. Rodchenko did not engage as actively in state projects as Lissitzky did. Whereas Lissitzky actually defined the practice of graphic and exhibition design in the 1930s, Rodchenko was marginalized by his primary creative community, photography, despite the fact that his work of the 1920s profoundly influenced work by other photographers who gained recognition in the Stalin years ${ }^{33}$."

Les questions de réalisme et de véracité photographiques, qui sont au cœur de notre étude, nous amène au constat suivant concernant la revue URSS en construction : à travers le photomontage, les éditeurs du magazine ne cherchaient-ils pas dès le départ à réécrire l'histoire de la construction de l'URSS ? Comme nous l'avons déjà mentionné, l'essence même de la revue repose sur le pouvoir évocateur de l'image qui surpasse celui des mots. Le lectorat, quelle que soit sa classe sociale

31. Alexandre Lavrentiev, Alexandre Rodchenko, Photographies 1924-1954, Paris, Gründ, 1995. 32. Voir David Elliott, David King, Alexander Rodchenko, Oxford, Oxford Museum of Modern Art, 1979.

33. « Lissitzky se prêtait avec énergie à toutes les commandes qu'on lui confiait, et en effet il devint le concepteur de publications et d'expositions le plus compétent de l'Union soviétique des années 1930. Rodchenko ne participait pas avec la même fréquence aux projets de l'État. Tandis que Lissitzky définissait l'art graphique et le design d'expositions des années 1930, Rodchenko était mis à l'écart par sa communauté artistique d'origine, celle des photographes, et ce malgré I'influence profonde de son travail des années 1920 sur d'autres photographes qui allaient accéder à la notoriété sous Staline. »Op. cit. note 7, p. 212. Traduction de I'auteur. 
ou sa nationalité, peut assister à l'histoire de l'URSS qui est en train de s'écrire devant ses yeux à travers les photographies. Mais de quelle histoire s'agit-il ? Est-ce l'histoire narrée du point de vue des éditeurs et qui tire davantage vers le mythe, ou de l'Histoire, la science humaine qui est le témoignage du passé dans sa forme la plus objective ? Où se place la photographie dans cette opposition entre histoire et Histoire?

Ce phénomène de réécriture historique par la photographie est intrinsèquement lié aux totalitarismes, car il implique le contrôle total de l'État sur les images produites sur son territoire et diffusées à l'extérieur comme à l'intérieur. On ne peut réécrire les événements si la liberté d'expression prévaut, car alors rien n'empêche un artiste de produire une photographie qui viendrait décrédibiliser la version officielle de l'Etat. En Union Soviétique, en raison des directives de 1932 interdisant toute société artistique indépendante ${ }^{34}$, la question de pluralité de voix photographiques ne se pose pas. Il n'existe qu'une version des faits, qu'un objectif et qu'une Histoire : celle que Staline a décidée et qui omet tout ce qui viendrait assombrir la gloire de son action au sein de la société soviétique. URSS en construction devient, pendant la plus grande partie de sa publication, l'incarnation de ce fantasme historique. Son succès en URSS, comme à l'étranger, est révélateur du pouvoir de l'image sur la perception de la réalité. Il est difficile de dire si le lectorat américain, anglais ou français est pleinement conscient de cet effet de réécriture ou même si cela lui importe en tant que spectateur extérieur, mais en souscrivant à la revue, il participe d'une certaine manière à son artifice.

Le développement du photomontage et l'invention d'un nouveau langage photographique industriel, ainsi que l'influence qu'il a sur l'art et sur la société, entraînent ces créateurs dans une spirale où la part de responsabilité dans les crimes commis par le Parti est estompée par la fascination de la création en construction. L'artiste ne veut plus ou ne peut plus avoir un regard critique sur les conséquences de sa collaboration avec l'État, d'autant que Rodchenko et Lissitzky ont toujours maintenu que le stalinisme n'était qu'une étape intermédiaire nécessaire à un communisme beaucoup plus libertaire. Pris dans un cercle à la fois vicieux et vertueux, ils ne sont plus totalement maittres de leur libre arbitre. Si cette réécriture est un acte conscient du gouvernement, il ne l'est pas complètement pour les photographes qui y ont contribué. Par désespoir, ou résignation, peutêtre se sont-ils pris fait prendre à leur propre jeu et se sont, eux-aussi, mis à croire à cette nouvelle Histoire de l'URSS, lavée de ses atrocités et ses torts.

\section{L'auteur}

Après un Bachelor Degree en histoire de l'art et en muséologie, et une thèse de fin d'études portant sur l'œuvre d'Alexej von Jawlensky au Smith College (Massachussetts, USA), Jessica Watson a soutenu en 2012 un mémoire d'étude sous la direction de Didier Schulmann et Rémi Parcollet consacré aux photographies de la Première Foire Internationale Dada à Berlin. En 2013, elle a rédigé un mémoire de recherche sous la direction de Dominique de Font-Réaulx et Michel Poivert autour de la revue photographique URSS en construction (1929-1949) et de ses enjeux éthiques et esthétiques. 